\title{
Severe Agitation in Dementia: An Explorative Secondary Data Analysis on the Prevalence and Associated Factors in Nursing Home Residents
}

\author{
Rebecca Palm ${ }^{\mathrm{a}, \mathrm{b}, *}$, Christian G.G. Sorg ${ }^{\mathrm{b}}$, Armin Ströbel ${ }^{\mathrm{a}}$, Debby L. Gerritsen ${ }^{\mathrm{c}}$ and Bernhard Holle ${ }^{\mathrm{a}, \mathrm{b}}$ \\ ${ }^{\mathrm{a}}$ German Center for Neurodegenerative Diseases (DZNE), Witten, Germany \\ ${ }^{\mathrm{b}}$ School of Nursing Science, University Witten/Herdecke, Witten, Germany \\ ${ }^{\mathrm{c}}$ Department of Primary and Community Care and Radboudumc Alzheimer Centre, Radboud University \\ Medical Centre, Nijmegen, The Netherlands
}

Accepted 3 October 2018

\begin{abstract}
.
Background: The phenomena of severe agitation is not well understood and often not adequately treated.

Objective: This article determines the prevalence and associated factors of severe agitation in nursing home residents with dementia.

Methods: Secondary data analysis within an observational study in German nursing homes with $n=1,967$ participants. We assessed severity of agitation with the Neuropsychiatric Inventory Questionnaire (NPI-Q) and defined the construct of agitation as a composite score of the NPI-Q items agitation/aggression, disinhibition, and irritability/lability; the dependent variable of severe agitation was considered as being present in residents who scored 'severe' in at least one of these symptoms. A binary logistic regression model was calculated to estimate associations.

Results: The prevalence of severe agitation was $6.3 \%(n=124)$. The strongest associations were found for elation/euphoria (OR 7.6, CI 3.1-18.5), delusions (OR 7.3, CI 4.0-13.2), apathy/indifference (OR 2.8, CI 1.7-4.7), anxiety (OR 2.2, CI 1.2-3.8), nighttime behaviors (OR 2.4, CI 1.4-4.2), motor disturbances (OR 2.4, CI 1.4-4.1), and male sex (OR 2.4. CI 1.3-4.2).

Conclusion: Severe agitation in nursing home residents with dementia is a relevant clinical issue as approximately $70 \%$ of residents have a dementia. Residents with elation/euphoria and delusions may have a stronger risk of showing severe agitation. We consider delusions as a possible cause of agitation and therefore a prelude to agitation. Although it might be possible that elation/euphoria follows from agitation, we hypothesize that the residents first experience elation/ euphoria and exhibit agitation afterwards.
\end{abstract}

Keywords: Aggression, agitation, dementia, nursing home, prevalence

\section{INTRODUCTION}

Agitation is recognized as a behavioral feature of neurocognitive disorders [1]. According to Cohen-

\footnotetext{
*Correspondence to: Rebecca Palm, PhD, German Center for Neurodegenerative Diseases (DZNE), Stockumer Straße 12, 58453 Witten, Germany. Tel.: +49 2302926 224; Fax: +49 2302926 239; E-mail: Rebecca.Palm@dzne.de.
}

Mansfield and Billig, agitation may also be expressed as verbally and physically nonaggressive behavior that needs to be distinguished from behavior that can clearly be explained by a need of the resident and by medical conditions that may provoke agitation [2]. In their definition, agitation is a perceived inappropriate behavior [2, 3]. In the recent definition from the International Psychogeriatric Association, 
patients with agitation persistently show at least one type of behavior that is associated with distress, such as excessive motor activity, verbal aggression, or physical aggression [4]. The existing classifications of agitation based on the Neuropsychiatric Inventory (NPI) include the items of agitation/aggression, disinhibition, and irritability/lability (and motor disturbance) [5-7]. The data indicate that agitation (including the items agitation/aggression, disinhibition, irritability/lability, and motor disturbance), with a weighted mean prevalence of $30 \%$, as measured by the NPI, is one of the neuropsychiatric subsyndromes that has the highest prevalence in nursing homes [6]. Among the symptoms of agitation in nursing home residents with dementia, the highest frequencies were reported for verbal nonaggressive behaviors, and the strongest ratings of disruptiveness were reported for verbal aggression [8]. The predictors of agitation have been identified as younger age, the male sex, and dementia type and stage [9-11]. Studies reporting on the association with determinants that are not resident-related, such as the nursing home environment, the type of care delivered, and social support, show inconsistent results [11].

Agitation implies distress for the residents, the informal caregivers, and the nurses. For residents with dementia, severe agitation may be associated with a higher use of psychotropic medication and physical restraints [9], as these interventions are generally applied as the last treatment options if other approaches remain ineffective [12]. Because of the side effects of psychotropic medications and the risks associated with physical restraints, residents who receive them show poorer health and care outcomes $[13,14]$. Agitation is also one of the most distressing symptoms for informal caregivers who care for their relatives [15]. Nurses in nursing homes show the highest distress scores for agitation compared to other neuropsychiatric symptoms; the score is predicted by the severity, but not by the frequency [16]. Because severe agitation may intensify the distress of professional caregivers [16], it needs to be considered as a risk factor for burnout, reduced workability, and generally weaker health [17].

Hardly any knowledge exists about the prevalence of and factors associated with severe agitation. A recent study reported on the prevalence and associated factors of very frequent agitation [18]; the authors emphasized the need for further studies exploring the relationship between different neuropsychiatric symptoms and agitation, as well as contextual factors in which agitation occurs in nursing home residents with dementia. This knowledge is needed to understand what triggers severe agitation and to develop interventions that prevent these behaviors in order to support nurses and caregivers in their daily work.

The objective of this article is to present the prevalence of severe agitation and its association to resident characteristics and other neuropsychiatric symptoms in a German sample of nursing home residents with dementia.

\section{METHODS}

\section{Study design}

The data for this secondary analysis were derived from the DemenzMonitor study, which had an observational, longitudinal design [19]. The study was conducted in a convenience sample of German nursing homes that were approved by the German long-term care insurance for providing skilled nursing care $24 / 7$. The nursing homes were recruited by public announcements in newsletters and journals. Residents who provided informed consent, or for whom informed consent was provided by their legal representatives, participated in the DemenzMonitor study. Participating nursing homes were encouraged to include all of their residents. Because participation was voluntarily, the nursing homes decided with how many of their organizational care units they wanted to participate.

The nursing homes that participated in the DemenzMonitor study were representative for German nursing homes with respect to the case mix of the residents' care dependency (as assessed by the statutory long term care insurance) and staff levels. Compared to average German nursing homes, the participating institutions were twice as large and more often provided Dementia Special Care Units than it is estimated for Germany (40\% versus $30 \%)$ [20].

For this analysis, we selected the data from the first measurement of residents who had a diagnosis of dementia in their records and were screened as positive for at least mild cognitive impairment at the time of measurement. We combined these two inclusion criteria to avoid the inclusion of false-positives because the recorded diagnosis is known to be unreliable [21]. The three datasets of the measurements from 2012, 2013, and 2014 were pooled and used as the dataset for analysis. 


\section{Procedure of data collection}

The data were collected by nursing home staff every year over one month (2012-2014). The researchers trained one staff member of each nursing home (study coordinator) on a one-day lecture in using the assessments. The study coordinators either assessed the residents themselves or they trained other nurses in performing the assessments. The assessments were to be performed by the nurse who was most familiar with the resident. On the resident level, they assessed the cognitive and physical function (self-care abilities) and neuropsychiatric symptoms of each participant; the nurses collected information on each participant's dementia diagnosis and sociodemographic data from the medical records of the residents. Sociodemographic data included age, sex, and existence of a court order to stay in the nursing home, length of stay, and frequency of visits from external visitors.

\section{Measurements}

The prevalence and severity of the neuropsychiatric symptoms were assessed with the NPI-Q, a retrospective, self-administered questionnaire that includes 12 symptom domains (1-mild, 2-moderate, and 3-severe within the prior month) and is completed by informants in regard to the patients for whom they care [22]. The NPI-Q is a short-form of the NPI-NH (nursing home) [23]. It assesses the items delusions, hallucinations, depression/dysphoria, anxiety, elation/ euphoria, apathy/indifference, motor disturbance, nighttime behaviors, and behavior related to appetite/eating. The latter item assesses if changes appeared regarding the appetite and eating behavior or if the resident experienced any changes in weight (in- or decrease).

Cognitive impairment was assessed with the Dementia Screening Scale (DSS), a seven-item proxy rating scale (range $0-14$, higher values indicate greater impairment), which was developed for use by nursing home staff and has been tested satisfactorily [24]. We applied the recommended cut-off score to identify participants with cognitive impairments (DSS > 2) [24]. Impairment in physical functions and self-care abilities was assessed with the Physical SelfMaintenance Scale (PSMS) [25].

As an indicator of social support, the nurses assessed how often each participant was visited by different groups of external visitors (family, friends, and neighbors) during the week prior. We calculated a visit score as follows: the number of external visitor groups $x$ the frequency of visits to residents during the week prior to the assessment (range 0-16).

Three indicators of the nursing home environment and the model of care were assessed: the size of the care unit, residence in a Dementia Care Unit and residence in a closed unit (where residents need legal consent to live there).

\section{Variables}

We defined the construct agitation as a composite score of the NPI-Q items agitation/aggression, disinhibition, and irritability/lability based on a previously conducted mokken analysis using the software $R$ and the package 'mokken' [26]. The mokken scaling analysis is a method of the non-parametric item response theory and a useful tool for researchers to find a relationship between items in scales [27]. The method is established in the context of the development of scales that comprise multiple binary or polytomous items, which is the case for the NPI-Q with four response categories. The coefficient Loevinger's H and Cronbach's alpha were applied to interpret the scalability and internal consistency of the NPI-Q subscales.

We defined three agitation categories: 1) Severe agitation is defined as a score of 3 in at least one of the three NPI scales agitation/aggression, disinhibition or irritability/lability; 2) No agitation is defined as a score of 0 in all three of these NPI items; and 3) Mild agitation: all remaining residents between these two categories.

We compared the categories 'severe agitation' with the category 'no agitation' to identify those variables associated with severe agitation.

We decided to exclude residents with mild agitation because we expected that the associated factors would be more distinct in the group of residents with severe agitation compared to residents with no symptoms of agitation.

To verify this assumption, we conducted a sensitivity analysis using the same variables and statistical procedures on the whole data set, i.e., category 'no agitation' versus category 'mild or severe agitation'. The main results for the comparison of these two groups are shown. The results of the sensitivity analysis are shown in the Supplementary Material.

\section{Data analysis}

We performed descriptive statistics to describe the differences between the comparison groups and tests 
of statistical significance ( $\chi^{2}$ and independent $t$-tests) to interpret the differences. Significant results were not interpreted with respect to the generalizability of results but rather in regard to select variables for the multivariate model. We included the variables age, sex, length of stay, living situation before residing in the nursing home, PSMS score, DSS score, and the presence of every NPI-Q item (except those used as dependent variables); we also included variables measured on the care unit level: residence in a closed unit, residence in a dementia care unit, and size of the care unit. Because the data are nested on three levels, we initially calculated a generalized linear mixed model with random effects for nursing home and care unit, but the random effects were not significant. We removed the random effects and calculated a binary logistic regression model. To verify our findings of this model we drew a random sample of our data (50\% without replication) and ran the same model. Additionally, we calculated the model in the stepwise forward method. The results of verification analysis pointed in the same direction as the reported model; therefore, these data are not shown.

\section{RESULTS}

During the measurements (2012-2014), the participating care units $(n=140)$ of the nursing homes provided 5,873 beds over three years (for $n=2$ care units data on bed capacity is missing; they are excluded in this figure). In the DemenzMonitor study, we collected $n=4,264$ questionnaires (participation rate of $72.6 \%$ ).

The total sample of the DemenzMonitor study consisted of 2,906 participants at their first measurement. Of these, 903 were excluded because no dementia diagnosis was recorded $(n=781)$ and/or because their DSS Score was $<3(n=527)$. Among the 903 excluded residents, a number of $n=122$ had a diagnosis of dementia in their records but did not screen positive for cognitive impairment at the same time. Vice versa, $n=376$ participants that screened positive for cognitive impairment had no dementia diagnosis at the same time. In addition, 36 had to be excluded because of missing or invalid NPI-Q data. The excluded participants were younger, more often male, had a higher visit score, had less cognitive and functional impairments, and lived less often in a (closed) Dementia Care Unit. Except the items depression/dysphoria and elation/euphoria, the excluded participants scored lower and less often on the NPI (see the complete results in Supplementary Table 1).

The analysis sample consisted of 1,967 participants.

In this sample, the prevalence of severe agitation was $6.3 \%(n=124)$; the mean severity was 5.6 (Min 3 , Max 9). Further, 51.2\% $(n=1,008)$ of the participants showed no symptoms of agitation. A group of 835 participants $(42.5 \%)$ showed mild-moderate agitation. The characteristics of residents with severe agitation and without agitation are described in Table 1.

The participants with severe agitation were more often male, younger, and more cognitively and functionally impaired than the participants without agitation. The groups differed also with respect to the length of stay in the nursing home, indicating that the participants with severe agitation had a shorter length of stay. The visit score did not differ between the groups.

The participants with severe agitation showed a higher prevalence and mean severity of all NPI-Q items (Table 1).

In the mokken analysis, our agitation subscale showed a medium scalability $(\mathrm{H}=0.46)$ [27] and a questionable internal consistency $(\alpha=0.69)$ [28], according to the recommended cut-offs. The results of the complete mokken analysis are shown in Supplementary Table 2.

In the regression model, we found statistically significant associations $(p \leq 0.001)$ between severe agitation and the variables delusions, elation/euphoria, and apathy/indifference. Elation/euphoria and delusions showed the strongest associations with severe agitation. The variables male sex, anxiety, motor disturbance, nighttime behaviors and appetite/eating were also statistically significant $(p<0.05)$. The other variables showed no statistically significant associations with severe agitation in the model (Table 2). The results of the sensitivity analysis pointed in the same direction, but all odds ratios were smaller compared to the main analysis (see Supplementary Table 3).

\section{DISCUSSION}

Our findings showed that severe agitation was present in 6 out of 100 nursing home residents with dementia. This finding was comparable to that of a previous study that showed a prevalence of $7.4 \%$ for very frequent agitation, as measured by the Cohen- 
Table 1

Characteristics of the study sample (values are reported as relative frequencies (prevalence) and the means (severity). NA - not applicable as the variables were used for the definition of severe agitation

\begin{tabular}{|c|c|c|c|c|c|}
\hline & $\mathrm{n}$ & Residents with severe agitation & $\mathrm{n}$ & Residents without severe agitation & $\mathrm{p}^{\mathrm{b}}$ \\
\hline \multicolumn{6}{|l|}{ Sociodemographics } \\
\hline Age $(y)$ & 124 & $80.8( \pm 10.1)$ & 1,008 & $83.7( \pm 7.6)$ & $<0.001$ \\
\hline Male sex & 124 & $40(32.3)$ & 1,008 & $196(19.4)$ & $<0.001$ \\
\hline Resident has a court order to stay in $\mathrm{NH}$ & 119 & $19(16.0)$ & 1,004 & $97(9.7)$ & 0.038 \\
\hline Length of stay (months) & 124 & $28.4( \pm 28.4)$ & 1,008 & $35.8( \pm 42.7)$ & 0.046 \\
\hline Frequency of visits (score $0-16)$ (mean) & 124 & $4.1( \pm 2.5)$ & 1,008 & $4.0( \pm 2.3)$ & 0.765 \\
\hline \multicolumn{6}{|l|}{ Dementia \& care dependency } \\
\hline Dementia Screening Scale $(3-14)^{\mathrm{a}}$ & 124 & 10.4 & 1,008 & 9.4 & $<0.001$ \\
\hline Physical Self-Maintenance Score $(6-30)^{\mathrm{a}}$ & 121 & 21.3 & 1,002 & 20.1 & 0.013 \\
\hline \multicolumn{6}{|l|}{ Nursing home environment and model of care } \\
\hline Lives in a Dementia Care Unit & 124 & $66(53.2)$ & 1,008 & $425(42.2)$ & 0.021 \\
\hline Lives in a closed unit & & $26(21.0)$ & & $135(13.4)$ & 0.029 \\
\hline Size of the care unit (places) & & $23.7( \pm 8.3)$ & & $27.1( \pm 9.5)$ & $<0.001$ \\
\hline Neuropsychiatric symptoms & & $\%$ (mean severity) & & $\%$ (mean severity) & \\
\hline Agitation/aggression & 124 & $81.5(2.1)$ & 1,008 & 0.0 & NA \\
\hline Disinhibition & & $63.7(1.5)$ & & 0.0 & NA \\
\hline Irritability/lability & & $78.2(2.0)$ & & 0.0 & NA \\
\hline Delusions & & $44.4(1.0)$ & & $6.8(0.1)$ & $<0.001$ \\
\hline Hallucinations & & $29.8(0.6)$ & & $8.9(0.1)$ & $<0.001$ \\
\hline Depression/dysphoria & & $45.2(0.9)$ & & $19.0(0.3)$ & $<0.001$ \\
\hline Anxiety & & $42.7(1.0)$ & & $12.0(0.2)$ & $<0.001$ \\
\hline Motor disturbance & & $62.1(1.4)$ & & $22.4(0.4)$ & $<0.001$ \\
\hline Nighttime behaviors & & $50.8(1.0)$ & & $15.6(0.2)$ & $<0.001$ \\
\hline Elation/euphoria & & $16.9(0.3)$ & & $2.6(0.0)$ & $<0.001$ \\
\hline Apathy/indifference & & $48.4(1.0)$ & & $19.7(0.3)$ & $<0.001$ \\
\hline Appetite/eating & & $46.0(0.9)$ & & $25.4(0.4)$ & $<0.001$ \\
\hline
\end{tabular}

${ }^{a}$ higher scores indicate greater impairment. ${ }^{\mathrm{b}}$ generated by $\chi^{2}$ test or an independent $t$-test.

Table 2

Results of the regression model

\begin{tabular}{lccc}
\hline Characteristics & Odds Ratio severe agitation versus no agitation & $\mathrm{CI}(95 \%)$ & $\mathrm{p}$ \\
\hline Age & 0.9 & $0.9-1.0$ & 0.159 \\
Male sex & $\mathbf{2 . 4}$ & $\mathbf{1 . 3}-\mathbf{4 . 2}$ & $\mathbf{0 . 0 0 2}$ \\
Length of stay (months) & 0.9 & $0.9-1.0$ & 0.094 \\
Resident has a court order to stay in NH & 1.9 & $0.9-4.0$ & 0.081 \\
Dementia Screening Scale & 1.0 & $0.9-1.1$ & 0.870 \\
Physical Self-Maintenance Score & 1.0 & $0.9-1.1$ & 0.148 \\
Lives in a Dementia Care Unit & 1.4 & $0.8-2.4$ & 0.192 \\
Lives in a closed unit & 1.6 & $0.7-3.4$ & 0.799 \\
Size of the care unit (places) & 0.9 & $0.9-1.0$ & 0.161 \\
Delusions & $\mathbf{7 . 3}$ & $\mathbf{4 . 0 - 1 3 . 2}$ & $<\mathbf{0 . 0 0 1}$ \\
Hallucinations & 1.3 & $0.6-25$ & 0.419 \\
Depression/ dysphoria & 1.5 & $0.9-2.7$ & 0.091 \\
Anxiety & $\mathbf{2 . 2}$ & $\mathbf{1 . 2 - 3 . 8}$ & $\mathbf{0 . 0 0 6}$ \\
Elation/euphoria & $\mathbf{7 . 6}$ & $\mathbf{3 . 1}-\mathbf{1 8 . 5}$ & $<\mathbf{0 . 0 0 1}$ \\
Apathy/indifference & $\mathbf{2 . 8}$ & $\mathbf{1 . 7}-\mathbf{4 . 7}$ & $<\mathbf{0 . 0 0 1}$ \\
Motor disturbance & $\mathbf{2 . 4}$ & $\mathbf{1 . 4 - 4 . 1}$ & $\mathbf{0 . 0 0 1}$ \\
Nighttime behaviors & $\mathbf{2 . 4}$ & $\mathbf{1 . 4 - 4 . 2}$ & $\mathbf{0 . 0 0 1}$ \\
Appetite/eating & 1.4 & $0.8-2.4$ & 0.128 \\
\hline
\end{tabular}

Participants included in the analysis: $n=1116$ Model fit: Cox \& Snell $\mathrm{R}^{2}=0.222$, Nagelkerkes $\mathrm{R}^{2}=0.452$.

Mansfield Agitation Inventory (CMAI) [18]. We also found that severe agitation was associated with the presence of other neuropsychiatric symptoms, a finding that was previously presented by van der Mussele et al. regarding agitation in general [29]. Of all the neuropsychiatric symptoms, elation/euphoria and delusions were identified as having the strongest associations with severe agitation. This finding was also in 
line with previous research that identified both symptoms as correlated factors of very frequent agitation, as measured by the CMAI [18].

We also confirmed the risk factor of the male sex for severe agitation that was previously found to be associated with agitation/aggression in a range of studies [9].

We also found that behavior related to the nighttime and eating/appetite was significantly associated with agitation. This finding underlines the importance of environmental factors that may cause agitation when the resident's needs are not met. If care practices in a nursing home are ignoring residents individual needs - such as basic needs like sleep and nutrition - this may lead to agitation [30].

We could not confirm the result that the severity of dementia is associated with severe agitation, as was found previously for agitation $[9,31]$ and for very frequent agitation [18]. This might be explained by the fact that the instrument we used to screen for dementia, the Dementia Screening Scale [24], did not provide a staging of dementia severity, which is provided by the Global Deterioration Scale that was used in both of the other studies.

We did not find that residents with severe agitation had less social support, as measured by the frequency of different visitors. Other studies revealed that residents who more often received visitors or phone calls or who more often communicated with staff showed less agitation [31]. The reason why we did not find an association between the number or frequency of visitors and severe agitation may be because we only considered visits from external persons. Associations with severe agitation may be better explained by social support that is provided by people who are affiliated with the nursing home and are more constantly present, or it could explained by the characteristics of the staff [32].

Structural factors of the nursing home environment, such as the size of the care unit and the concept (dementia care units versus traditional care unit), were not associated with severe agitation in our study. Previous research revealed different results regarding the association between contextual factors and agitation [31]. A recent study from Switzerland showed that staff from dementia special care units experience aggression more often than staff in other care units [32]. The finding that severe agitation may not be associated with the nursing home environment or with the model of care in German nursing homes needs to be investigated more thoroughly. The dichotomous variables that we used to divide dementia care units and traditional care units in this study did not capture the heterogeneity and diversity of the care units and allowed only heuristic conclusions.

The limitations of our study have to be considered when interpreting our results. The instrument that we used to measure agitation, the NPI-Q, does not provide information on the frequency of the symptoms. This information would provide the possibility of investigating the relationship between the frequency and severity of severe agitation, which is needed to understand the similarity between the results from our study and those of the study by VeldwijkRouwenhorst and colleagues [18]. However, studies show that there is a strong relationship between frequency and severity of NPI symptoms [22]. But the examples that are given to illustrate the NPI-Q items are much shorter compared to the original version, the NPI-NH [23]; this may have influenced the assessment and could explain the differences found in our results compared to those of other studies that used the NPI-NH. In our study the researchers were not able to perform clinical assessments but had to rely on the nurses' observational assessments. The data of observational assessments may be of lesser quality than that of clinical assessments. Because we did not perform clinical assessments to evaluate the dementia diagnosis we combined the diagnosis in the records with a proxy-rated assessment of cognition. If longitudinal data were available for one resident, we checked if the diagnosis was recorded repeatedly or there was a mistake in the records. However, we may have excluded residents with dementia if their diagnosis was not recorded properly.

When interpreting the results from this study, one must keep in mind that the phenomenon of agitation is defined differently across studies and that the symptoms may overlap with those from other concepts such as aggression. This may also explain why the scalability of the construct agitation does not show strong results. Another important issue that needs to be considered is the fact that agitation is associated with other determinants that we did not assess and could not include in our analysis (e.g., pain and psychotropic medication) $[10,11]$. Further, our results did not rely on longitudinal data; hence, we were not able to provide evidence for the direction of the investigated phenomena. Severe agitation may occur as a reaction to the environment if the environment does not respond adequately to delusions and elation/euphoria. The results from this study may not be generalizable, because the nursing homes that 
participated were larger than the average nursing home. As environmental attributes may correlate with behavior, the size of the nursing home may play a role.

Despite these limitations, this study had several strengths. The short screening instruments (like the NPI-Q or the DSS) were feasible to administer to a large number of residents from several nursing homes; this allowed us to collect data from a large sample of residents, which are needed to investigate singular conditions. Therefore, we did not need to match datasets from different studies, which, in turn, have other limitations [18]. We investigated the severity of agitation as a dependent variable which may be more important than frequency, because nurses are more strongly distressed by the severity of neuropsychiatric symptoms [16].

We concluded that delusions and elation/euphoria seem to be risk factors for severe agitation in nursing home residents with dementia. Both symptoms also seemed to be related in terms of the frequency of agitation [18]. We hypothesize that delusions and elation/euphoria precede severe agitation and suggest further research to test this hypothesis. Therefore, a broad understanding of these two phenomena in the context of dementia needs to be developed. Regarding delusions, a prevailing classification of the different types is available [33]. The authors describe environmental factors, personal factors and the dementia itself as reasons underlying delusions in dementia. It would be of interest to investigate which of these factors are particularly associated with severe agitation and the role that the reactions of the staff play in the development of severe agitation. A differentiation of reasons underlying delusions in dementia is also needed to distinguish clearly if the associated behavior should really be classified as agitation as it is understood by Cohen-Mansfield and Billig [3] or if its causes are clear so that it can be considered as a behavior that evidently is a reaction to needs or confusion.

Regarding elation/euphoria, one must keep in mind that this condition occurs infrequently, even in the group of residents with severe agitation. Therefore, it may be of limited clinical relevance. However, to understand this concept and its association with severe agitation, an in-depth analysis of its appearance and causes is required. Such an examination would be of value for conceptual knowledge on neuropsychiatric symptoms and for developing interventions for support staff to prevent and manage severe agitation in dementia.
We concluded that further studies are needed to investigate the association and potential causal relationships among delusions, euphoria and severe agitation. Therefore, each concept needs to be assessed with instruments that capture the respective domains. A prospective analysis with longitudinal data might be the best choice to explain the causes of severe agitation.

\section{ACKNOWLEDGMENTS}

We thank Sven Reuther, Johannes Bergmann and Diana Trutschel for reviewing the manuscript before submission and giving us a constructive feedback.

Authors' disclosures available online (https:// www.j-alz.com/manuscript-disclosures/18-0647r2).

\section{SUPPLEMENTARY MATERIAL}

The supplementary material is available in the electronic version of this article: http://dx.doi.org/ 10.3233/JAD-180647.

\section{REFERENCES}

[1] American Psychiatric Association (2013) DSM-5. Diagnostic and statistical manual of mental disorders, American Psychiatric Publishing, Washington, DC.

[2] Cohen-Mansfield J (1996) Conceptualization of agitation: Results based on the Cohen-Mansfield Agitation Inventory and the Agitation Behavior Mapping Instrument. Int Psychogeriatr 8(Suppl 3), 309-315; discussion 351-304.

[3] Cohen-Mansfield J, Billig N (1986) Agitated behaviors in the elderly. I. A conceptual review. J Am Geriatr Soc 34, 711-721.

[4] Cummings J, Mintzer J, Brodaty H, Sano M, Banerjee S, Devanand DP, Gauthier S, Howard R, Lanctot K, Lyketsos CG, Peskind E, Porsteinsson AP, Reich E, Sampaio C, Steffens D, Wortmann M, Zhong K (2015) Agitation in cognitive disorders: International Psychogeriatric Association provisional consensus clinical and research definition. Int Psychogeriatr 27, 7-17.

[5] Selbaek G, Engedal K (2012) Stability of the factor structure of the Neuropsychiatric Inventory in a 31-month followup study of a large sample of nursing-home patients with dementia. Int Psychogeriatr 24, 62-73.

[6] Selbaek G, Engedal K, Bergh S (2013) The prevalence and course of neuropsychiatric symptoms in nursing home patients with dementia: A systematic review. J Am Med Dir Assoc 14, 161-169.

[7] Reuther S, Dichter MN, Bartholomeyczik S, Nordheim J, Halek M (2016) Construct validity and internal consistency of the neuropsychiatric inventory - nursing home (NPI-NH) in German nursing homes. Int Psychogeriatr 28, 1017-1027.

[8] Cohen-Mansfield J (2008) Agitated behavior in persons with dementia: The relationship between type of behavior, its frequency, and its disruptiveness. J Psychiatr Res $\mathbf{4 3}$, 64-69. 
[9] Zuidema S, Koopmans R, Verhey F (2007) Prevalence and predictors of neuropsychiatric symptoms in cognitively impaired nursing home patients. J Geriatr Psychiatry Neurol 20, 41-49.

[10] Livingston G, Barber J, Marston L, Rapaport P, Livingston D, Cousins S, Robertson S, La Frenais F, Cooper C (2017) Prevalence of and associations with agitation in residents with dementia living in care homes: MARQUE crosssectional study. BJPsych Open 3, 171-178.

[11] Kolanowski A, Boltz M, Galik E, Gitlin LN, Kales HC, Resnick B, Van Haitsma KS, Knehans A, Sutterlin JE, Sefcik JS, Liu W, Petrovsky DV, Massimo L, GilmoreBykovskyi A, MacAndrew M, Brewster G, Nalls V, Jao YL, Duffort N, Scerpella D (2017) Determinants of behavioral and psychological symptoms of dementia: A scoping review of the evidence. Nurs Outlook 65, 515-529.

[12] Ostaszkiewicz J, Lakhan P, O'Connell B, Hawkins M (2015) Ongoing challenges responding to behavioural and psychological symptoms of dementia. Int Nurs Rev 62, 506-516.

[13] Chiu Y, Bero L, Hessol NA, Lexchin J, Harrington C (2015) A literature review of clinical outcomes associated with antipsychotic medication use in North American nursing home residents. Health Policy 119, 802-813.

[14] Hofmann H, Hahn S (2014) Characteristics of nursing home residents and physical restraint: A systematic literature review. J Clin Nurs 23, 3012-3024.

[15] Feast A, Moniz-Cook E, Stoner C, Charlesworth G, Orrell M (2016) A systematic review of the relationship between behavioral and psychological symptoms (BPSD) and caregiver well-being. Int Psychogeriatr 28, 1761-1774.

[16] Zwijsen SA, Kabboord A, Eefsting JA, Hertogh CM, Pot AM, Gerritsen DL, Smalbrugge M (2014) Nurses in distress? An explorative study into the relation between distress and individual neuropsychiatric symptoms of people with dementia in nursing homes. Int J Geriatr Psychiatry 29, 384-391.

[17] Schmidt SG, Dichter MN, Palm R, Hasselhorn HM (2012) Distress experienced by nurses in response to the challenging behaviour of residents - evidence from German nursing homes. J Clin Nurs 21, 3134-3142.

[18] Veldwijk-Rouwenhorst AE, Smalbrugge M, Wetzels R, Bor H, Zuidema SU, Koopmans R, Gerritsen DL (2017) Nursing home residents with dementia and very frequent agitation: A particular group. Am J Geriatr Psychiatry 25, 1339-1348.

[19] Palm R, Köhler K, Schwab CG, Bartholomeyczik S, Holle B (2013) Longitudinal evaluation of dementia care in German nursing homes: The "DemenzMonitor" study protocol. BMC Geriatr 13, 123.

[20] Palm R, Holle B (2016) Forschungsbericht der Studie DemenzMonitor. https://www.dzne.de/fileadmin/user_up load/editors/images/Standorte/Witten/Projekte/DemenzMo nitor/2016_Forschungsbericht_DemenzMonitor_final. pdf, Witten.
[21] Palm R, Jünger S, Reuther S, Schwab CG, Dichter MN, Holle B, Halek M (2016) People with dementia in nursing home research: A methodological review of the definition and identification of the study population. BMC Geriatr 16, 78.

[22] Kaufer DI, Cummings JL, Ketchel P, Smith V, MacMillan A, Shelley T, Lopez OL, DeKosky ST (2000) Validation of the NPI-Q, a brief clinical form of the Neuropsychiatric Inventory. J Neuropsychiatry Clin Neurosci 12, 233-239.

[23] Cummings JL, Mega M, Gray K, Rosenberg-Thompson S, Carusi DA, Gornbein J (1994) The Neuropsychiatric Inventory: Comprehensive assessment of psychopathology in dementia. Neurology 44, 2308-2314.

[24] Köhler L, Weyerer S, Schaufele M (2007) Proxy screening tools improve the recognition of dementia in old-age homes: Results of a validation study. Age Ageing 36, 549-554.

[25] Lawton MP, Brody EM (1969) Assessment of older people: Self-maintaining and instrumental activities of daily living. Gerontologist 9, 179-186.

[26] Van der Ark L (2007) Mokken scale analysis in R. J Stat Softw 20. doi: 10.18637/jss.v020.i11

[27] Watson R, van der Ark LA, Lin LC, Fieo R, Deary IJ, Meijer RR (2011) Item response theory: How Mokken scaling can be used in clinical practice. J Clin Nurs 21, 2736-2746.

[28] Streiner DL (2003) Starting at the beginning: An introduction to coefficient alpha and internal consistency. J Pers Assess 80, 99-103.

[29] Van der Mussele S, Le Bastard N, Saerens J, Somers N, Marien P, Goeman J, De Deyn PP, Engelborghs S (2015) Agitation-associated behavioral symptoms in mild cognitive impairment and Alzheimer's dementia. Aging Ment Health 19, 247-257.

[30] Corbett A, Ballard C, Creese B (2017) Evidence-based management of behavioural and psychological symptoms of dementia. In Oxford Textbook of Geriatric Medicine, Michel JB, Beattie L, Finbarr MC, Walston JD, eds. Oxford University Press, Oxford, Section 12.

[31] Zuidema SU, de Jonghe JF, Verhey FR, Koopmans RT (2009) Predictors of neuropsychiatric symptoms in nursing home patients: Influence of gender and dementia severity. Int J Geriatr Psychiatry 24, 1079-1086.

[32] Stutte K, Hahn S, Fierz K, Zuniga F (2017) Factors associated with aggressive behavior between residents and staff in nursing homes. Geriatr Nurs 38, 398-405.

[33] Cohen-Mansfield J, Golander H, Cohen R (2017) Rethinking psychosis in dementia: An analysis of antecedents and explanations. Am J Alzheimers Dis Other Demen 32, 265-271. 